\title{
Recuperación de valor con harvester aplicando tecnología de optimización de trozado
}

\author{
Value recovery with harvester applying bucking optimization \\ technology.
}

\author{
Dionel Kimmich ${ }^{1}$, Eduardo Hildt ${ }^{{ }^{*}}$ e Patricio Miguel Mac Donagh ${ }^{1}$
}

\begin{abstract}
Summary
The on-board bucking optimization is a technology that makes possible add value to the harvested wood. In this study, it was analyzed the potential optimization of the harvested trees value and the harvester operational costs in Pinus taeda and Pinus elliottii var. elliottii x Pinus caribaea var. hondurensis (hybrid pine) plantations. Using StanForD files and digital videos, a times study was carried out to quantify the harvested wood value and the effective productivity of a mechanized thinning. It was found that the optimizer result in a $2 \%$ gain on the value of the harvested wood, and that the shape of the hybrid pine shaft favors the generation of value and improves the productivity of the harvester. A difference in income before taxes of $4.8 \%$ was observed between species and cutting technologies.
\end{abstract}

Keywords: Productivity, StanForD, Value generation.

\section{Resumen}

La optimización de trozado a bordo de harvesters es una tecnología que posibilita incrementar el valor de la madera cosechada. En este estudio se analizó el potencial de optimización del valor de los árboles cosechados y el costo de operación de un harvester en segundos raleos de Pinus taeda y en Pinus elliottii var. elliottii x Pinus caribaea var. hondurensis (Pino híbrido). Utilizando archivos StanForD y videos digitales, se realizó un estudio de tiempos y movimientos para cuantificar el impacto de la optimización sobre el valor de la madera elaborada y la productividad efectiva del raleo mecanizado. Se encontró que el optimizador genera una ganancia del $2 \%$ sobre el valor de la madera cosechada, y que además la forma del fuste del Pino híbrido favorece la generación de valor y mejora la productividad del harvester. Se observó una diferencia en el ingreso antes de impuestos del 4,8\% entre especies y tecnologías de trozado.

Palabras clave: Productividad, StanForD, Generación de valor.

\section{INTRODUCCIÓN}

La optimización del trozado de los árboles es una de las herramientas más promisorias para maximizar el valor de los bosques. Los harvesters modernos están provistos de computadoras a bordo que ejecutan modelos de optimización mientras se realiza la operación de cosecha, prediciendo el perfil del árbol cosechado y asistiendo al operario en la selección del producto óptimo a cortar (LABELLE; BERGEN.; WINDISCH, 2017; LU et al., 2018).

Las operaciones de raleos llevadas a cabo en la provincia de Misiones se caracterizan por un bajo volumen individual de los árboles, que condiciona la productividad del harvester y provoca márgenes de ganancia ajustados. La mejora constante de la productividad y el conocimiento de los factores que la afectan son importantes para asegurar la rentabilidad de los raleos mecanizados. En estudios locales realizados en raleos de Pinus taeda L., se midieron productividades de $12 \mathrm{~m}^{3} / \mathrm{h}$ para árboles de $0,1 \mathrm{~m}^{3}$ hasta $24 \mathrm{~m}^{3} / \mathrm{h}$ en árboles de $0,34 \mathrm{~m}^{3}$, y costos de 4,8 a 2,6 US $\$ / \mathrm{m}^{3}$ respectivamente. (MAC DONAGH et al., 2013a;b).

A nivel mundial, generalmente existe una diferenciación de precios de los rollos en función de tres factores principales, diámetro en punta fina, largo y tipificación por calidad (MALINEN et al., 2010; NAKAHATA et al., 2014). Los precios para rollos de Pinus sylvestris L. en dimensiones de $14-20 \mathrm{~cm}$ 
punta fina en Finlandia según Malinen et al., (2010), varían entre 53 - 58 US $\$ / \mathrm{m}^{3}$. En Australia, en un estudio llevado a cabo por Murphy et al., (2010), para P. radiata los precios para rollos aserrables de $15,5 \mathrm{~cm}$ en punta fina variaron de 68,7 a $83,6 \mathrm{US} \$ / \mathrm{m}^{3}$, al incrementarse su largo de $3,8 \mathrm{~m}$ a $5,5 \mathrm{~m}$. Sin embargo, en el Noreste Argentino, las industrias locales discriminan los precios únicamente en función del diámetro en punta fina, sin adoptar otra diferenciación por calidad o largo para la cotización de la madera en rollos.

Walsh (2012), en un estudio realizado en una tala rasa de Pinus radiata, con 2 parcelas de 100 árboles cada una, evaluó el uso de un optimizador de trozado a bordo de un harvester y determinó su productividad. Concluyó que el harvester con optimizador redujo los costos de cosecha por haber incrementado $9 \%$ la productividad y se generó un incremento de 3\% en el valor de la madera cosechada. Por otra parte, Akay (2017) reportó in incremento del 8,7\% en el valor de los árboles al aplicar un algoritmo de optimización de trozado, observando un mayor incremento de valor para los productos de mayor largo $(>5,5 \mathrm{~m})$ y las clases de diámetro intermedias $(30-39 \mathrm{~cm})$.

Labelle y Huß (2018), evaluando un harvester con optimizador en bosques de Picea abies L., encontraron que la optimización genera un ingreso 4\% mayor e incrementa la productividad del harvester en un 12,4\%. En cambio, Labelle, Bergen e Windisch, (2017), evaluaron las diferencias existentes entre el trozado automático optimizado y el trozado asistido por el operario, en una operación de cosecha de Pinus sylvestris L. Concluyeron que el trozado optimizado no afectó significativamente a la productividad ni incrementó el volumen cosechado. Por otra parte, debido a la variabilidad en la forma de los árboles, el trozado manual generó un incremento del 2,2\% en el valor de la madera para árboles con un diámetro mayor a $30 \mathrm{~cm}$. Estos resultados contrapuestos sugieren que el impacto de la optimización del trozado depende de las características del fuste y la copa de las distintas especies cosechadas.

El objetivo de este trabajo fue evaluar el potencial de generación de valor de un optimizador de trozado a bordo de un harvester en operaciones de segundo raleo, cuantificando su efecto sobre el fuste del híbrido Pinus elliottii var. elliottii x Pinus caribaea var. Hondurensis (Pino hibrido) respecto del $P$. taeda, evaluando además su efecto sobre la productividad y el costo de cosecha.

\section{MATERIALES Y MÉTODOS}

\section{Descripción del área de estudio}

La investigación se llevó a cabo en la zona norte de la Provincia de Misiones, localidad de Puerto Esperanza, Departamento de Iguazú. El suelo en ambos sitios es del tipo rojo profundo clasificado como "Kandiudult", según Soil Survey Staff (1999). El relieve de los predios es suavemente ondulado con pendiente una máxima de $4 \%$ y un promedio de 2,7\% en los rodales estudiados. El clima de la región se caracteriza por una temperatura media anual de $21^{\circ} \mathrm{C}$ y precipitaciones de alrededor de $2000 \mathrm{~mm}$ anuales, con régimen isohigro (PEÑA ZUBIATE; PINEDO, 1990). Los ensayos se ubicaron en rodales de Pinus taeda ( $26^{\circ} 5^{\prime}$ 59,39" Lat. S 54 35' 3,23" Long. O) y Pino híbrido ( $26^{\circ} 3^{\prime} 31,04^{\prime \prime}$ Lat. S $54^{\circ} 33^{\prime} 12,79^{\prime \prime}$ Long. O) sometidos a operaciones de segundo raleo.

El sistema de cosecha adoptado por la empresa contratista es el de corte a medida o "cut to length" (CTL) en inglés. Cada parcela consta de una calle por donde transita el harvester mientras ralea de forma selectiva 2 líneos a ambos lados. Los árboles cosechados fueron marcados previamente por la empresa propietaria de la plantación. Los criterios de marcación tuvieron como objetivo la extracción de los árboles bifurcados, con mala forma y con diámetros pequeños. El objetivo final de esta operación de raleo fue remover el $40 \%$ árboles.

\section{Descripción de la máquina evaluada}

El harvester que realizó la cosecha fue una máquina adaptada (SEIXAS; BATISTA, 2014). La máquina base fue una excavadora Hyundai ${ }^{\circledR} 160$ lc-7 de $94 \mathrm{~kW}$ de potencia, con una capacidad hidráulica de $2 \times 168$ l/min. El cabezal de cosecha fue de marca Kesla ${ }^{\circledR}$, modelo 25 RHS mkII, utilizando un sistema de control Motomit ${ }^{\circledR}$ IT con software de control versión "J" con asistente de optimización de trozado. Este harvester fue operado por un único operario de 27 años y con 3000 horas de experiencia en la realización de raleos. 
Kimmich, Hildt e Mac Donagh. - Recuperación de valor con harvester

aplicando tecnología de optimización de trozado

\section{Diseño del experimento y análisis estadístico.}

Las unidades experimentales fueron parcelas de 1537,5 m² (15 m x 102,5 m). Se realizó una prueba piloto para determinar el número mínimo de parcelas necesarias para obtener un error de muestreo máximo del 5\% con un 95\% de confianza, resultando en un mínimo de 7 parcelas por tratamiento.

Se utilizó un diseño experimental completamente al azar, con un arreglo factorial con 2 factores. El factor especie con las 2 especies antes nombradas y el factor optimización, con la aplicación o no del optimizador de trozado, resultando 4 tratamientos: $P$. taeda con optimización (P. taeda OPT ON), P. taeda sin optimización (P. taeda OPT OFF), Pino híbrido con optimización (P. híbrido OPT ON), Pino híbrido sin optimización (P. híbrido OPT OFF). Fueron medidas un total de 40 parcelas de 1537,5 m², 20 para cada especie evaluada. Los niveles de optimización se repartieron al azar entre las parcelas de cada especie, dando un total de 10 parcelas por tratamiento.

El optimizador del harvester fue configurado únicamente con una matriz de precios por producto, sin establecer limitaciones en las cantidades obtenidas para cada tipo de troza ("bucking to value") (UUSITALO, 2010). El trozado sin optimización consistió en otorgarle al operario la misma variedad de productos que fue configurada en la matriz de precio-producto del optimizador, pero desactivando la optimización y dejando completamente a su criterio la decisión del trozado. Esta libertad de decisión sobre el trozado es la forma de trabajo normal a la que el operario estaba acostumbrado y la más extendida en la región. El sistema de optimización de trozado del harvester fue programado con una gama de productos comúnmente demandados por las industrias y precios de mercado local en dólares (Tasa de cambio según BNA 14/07/2016 1 US\$ = 15 ARS\$) (Tabla 1).

En el análisis estadístico se consideró al volumen individual de los árboles cosechados como covariable, para independizar el efecto de los factores analizados respecto del efecto del volumen individual. Se realizó un análisis de covarianza (ANCOVA) para probar el efecto de la optimización de trozado en el valor por metro cúbico total (US $\$ / \mathrm{m}^{3} \mathrm{cc}$ ), y en el valor por volumen de madera aserrable total aprovechada (US $\$ / \mathrm{m}^{3}$ as $\mathrm{cc}$ ). También se evaluó su efecto en la productividad efectiva (PEF $\mathrm{m}^{3} \mathrm{cc} / \mathrm{h}$. ef.), costos del harvester y margen antes de impuestos en (US $\$ / \mathrm{m}^{3} \mathrm{cc}$ ). Para la determinación de diferencias significativas entre los factores analizados se realizó mediante el Test HSD de Tukey, con un nivel se significancia $\alpha=0,05$.

\section{Colección de datos}

Los datos de tiempos y movimientos para el análisis de productividad fueron generados mediante la grabación en video de la operación del harvester durante el ensayo. La cámara de video fue instalada en el interior de la cabina de tal forma que pudo registrar simultáneamente los datos del monitor de

Tabla 1 - Matriz de precios-producto aplicada en el sistema de control de harvester y comparación de precios de madera entre países en US\$.

Table 1 - Price-products matrix set on the harvester control system and comparison of average wood prices between countries in US\$.

\begin{tabular}{|c|c|c|c|c|}
\hline \multirow[b]{3}{*}{ Largos } & \multicolumn{4}{|c|}{ Precios aplicados en el sistema de control del harvester } \\
\hline & \multicolumn{4}{|c|}{ Diámetros en punta fina } \\
\hline & $14-18 \mathrm{~cm}$ & $18-25 \mathrm{~cm}$ & $25+\mathrm{cm}$ & Trozas pulpables \\
\hline $250 \mathrm{~cm}$ & 21,67 & 29,00 & 32,00 & 15,33 \\
\hline $315 \mathrm{~cm}$ & 21,67 & 29,00 & 32,00 & 15,33 \\
\hline \multirow[t]{5}{*}{$375 \mathrm{~cm}$} & 21,67 & 29,00 & 32,00 & 15,33 \\
\hline & \multicolumn{4}{|c|}{ Comparaciones de precios promedio (US\$) } \\
\hline & Finlandia & Nueva Zelanda & Estados Unidos & Argentina \\
\hline & P. sylvestris & P. radiata & P. taeda & P. taeda \\
\hline & $14-25 \mathrm{~cm}$ & $16-35 \mathrm{~cm}$ & $14-25 \mathrm{~cm}$ & $14-25 \mathrm{~cm}$ \\
\hline $250-270$ & 60 & 73 & 20 & 23 \\
\hline $300-340$ & 62 & 83 & 22 & 23 \\
\hline $340-375$ & 63 & 87 & 23 & 23 \\
\hline $375-400$ & 67 & 88 & 27 & 23 \\
\hline $400-500$ & 68 & 115 & 30 & -- \\
\hline $500+$ & -- & 115 & -- & -- \\
\hline
\end{tabular}

Fuente: Elaboración Propia. 
cosecha y los movimientos del cabezal cosechador. Cada ciclo operacional constó de cuatro actividades: búsqueda de árbol, volteo, trozado y liberación (Tabla 2). También se contabilizaron los tiempos de paradas productivas y tiempos de vuelta a " 0 " en los casos en que el operario necesitaba reiniciar la medición de longitud. Para analizar los videos se utilizó una herramienta basada en Visual Basic ${ }^{\circledR}$ para Microsoft Excel ${ }^{\circledR}$, que permitió capturar pantallas e identificar las actividades operacionales y registrar los tiempos de cada ciclo (NIEMISTÖ et al., 2012).

Tabla 2 - Descripción de las actividades del ciclo operacional

Table 2 - Operational cycle activities description

\begin{tabular}{|c|c|}
\hline Actividades del Ciclo & Descripción \\
\hline Búsqueda & $\begin{array}{l}\text { Movimiento de la máquina y la grúa desde la liberación de las ramas hasta el inicio del } \\
\text { corte de un nuevo árbol. }\end{array}$ \\
\hline Volteo & $\begin{array}{l}\text { Tiempo desde la activación de la espada de corte hasta el inicio de la alimentación del } \\
\text { cabezal con la primera troza. }\end{array}$ \\
\hline Trozado & $\begin{array}{l}\text { Tiempo de procesamiento del fuste, comprendiendo el desrame, medición y corte de los } \\
\text { productos. }\end{array}$ \\
\hline Liberación & $\begin{array}{l}\text { Tiempo que trascurre entre el corte de la última pieza y el desprendimiento de las ramas de la } \\
\text { copa. }\end{array}$ \\
\hline Parada productiva & $\begin{array}{l}\text { Tiempos contabilizados en actividades de movimiento de ramas, rollos o limpieza de } \\
\text { sotobosque. }\end{array}$ \\
\hline Vuelta a "0" & Tiempo requerido para poner a 0 el sensor de longitud del cabezal. \\
\hline
\end{tabular}

Fuente: Elaboración propia.

Los datos de los árboles cosechados por el harvester se extrajeron de la memoria auxiliar del sistema de control, en archivos StanForD ".stm" (Standard for Forest machine Data and Communication) (SKOGFORSK, 2016). Estos datos comprenden el número de trozas cortadas, el volumen por troza, los diámetros del fuste cada $50 \mathrm{~cm}$, el diámetro en punta fina (dpf), el volumen y tipo de producto de cada troza, tiempos efectivos y tiempo total de trabajo.

Para estimar con mayor precisión las características dasométricas de los rodales cosechados, se realizó un censo de los ensayos. Se midió el diámetro a la altura del pecho (DAP) de todos los árboles y se modelaron relaciones hipsométricas de árboles remanentes y cosechados con el fin de poder calcular datos pre-cosecha, post cosecha y la población cosechada.

\section{Análisis del costo}

Para realizar el cálculo de costo horario y costo de producción se siguió la metodología propuesta por Ackerman et al., (2014). Los precios de los insumos, valor de adquisición, antigüedad, vida útil, reparaciones, consumo de combustible, lubricantes, cadenas, salario, cargas sociales y elementos de protección personal de los operarios fueron proporcionados por la empresa contratista Albura S.A. Estos costos se presentan en dólares estadounidenses según la cotización del Banco de la Nación Argentina (tasa de cambio según BNA 14/07/2016 1 US\$ = 15 ARS\$).

\section{RESULTADOS Y DISCUSIÓN}

En este estudio se cosecharon un total $634,6 \mathrm{~m}^{3}$ de madera en rollos, provenientes de la intervención de 6,15 ha divididas en 2 ensayos, uno para $P$. taeda y otro para P. híbrido.

El rodal de P. taeda tuvo un mayor DAP y una menor variabilidad que el de P. híbrido, con un coeficiente de variación (C.V.) del DAP de 15,24\% para P. taeda y 19,74\% para P. híbrido. También la intensidad de raleo fue menor en P. híbrido, con 7,39 $\mathrm{m}^{2} /$ ha respecto de $P$. taeda con $10,40 \mathrm{~m}^{2} / \mathrm{ha}$. Otro parámetro destacable es que el rodal de P. taeda tuvo una mayor densidad (área basal) que el de P. híbrido antes de la intervención: 33,01 $\mathrm{m}^{2} /$ ha y $30,01 \mathrm{~m}^{2} /$ ha respectivamente. Estos dos últimos parámetros determinaron un mayor volumen de cosecha por parcela para $P$. taeda que para P. híbrido (Tabla 3).

En ambas especies se cosecharon un número similar de árboles, 961 para $P$. taeda y 902 para P. híbrido. El volumen individual medio de los árboles cosechados resultó un $9,37 \%$ superior en $P$. taeda respecto de P. híbrido, ambos con un desvío estándar similar de $0,15 \mathrm{~m}^{3}$ y $0,14 \mathrm{~m}^{3}$ respectivamente. 
Tabla 3 - Principales datos dasométricos de las plantaciones cosechadas.

Table 3 - Main dasometric data of harvested plantations.

\begin{tabular}{|c|c|c|c|c|c|c|c|c|}
\hline P. híbrido & $\frac{\mathrm{DAP}(\mathrm{cm})}{ \pm \mathrm{DE}}$ & $\begin{array}{l}\text { DAP } \\
\text { Mín }\end{array}$ & $\begin{array}{l}\text { DAP } \\
\text { Máx }\end{array}$ & $\frac{\text { Altura }(m)}{ \pm D E}$ & $\frac{\text { Densidad }}{\text { (árb/ha) }}$ & $A B\left(m^{2} / h a\right)$ & $\begin{array}{l}\text { Proporción } \\
\text { (\% árb/ha) }\end{array}$ & VOLi $\left(m^{3}\right)$ \\
\hline Post-cosecha & $26,4 b \pm 3,68$ & 16 & 41,0 & & 404 & 22,62 & $66 \%$ & $0,490 \mathrm{~b}$ \\
\hline Árboles cosechados & $20,6 b \pm 4,07$ & 8 & 38,5 & & 212 & 7,38 & $34 \%$ & $0,301 \mathrm{~b}$ \\
\hline Pre-cosecha & $24,4 b \pm 4,71$ & 8 & 41,0 & $20,3 \pm 2,05$ & 616 & 30,00 & $100 \%$ & $0,420 \mathrm{~b}$ \\
\hline \multicolumn{9}{|l|}{ P. taeda } \\
\hline Post-cosecha & $27,7 a \pm 4,02$ & 18 & 46,5 & & 367 & 22,60 & $56 \%$ & $0,620 a$ \\
\hline Árboles cosechados & $21,2 a \pm 3,24$ & 10 & 33,8 & & 286 & 10,40 & $43 \%$ & $0,325 a$ \\
\hline Pre-cosecha & $24,8 a \pm 4,90$ & 10 & 46,5 & $19,7 \pm 1,48$ & 653 & 33,00 & $100 \%$ & $0,550 \mathrm{a}$ \\
\hline
\end{tabular}

DAP: Diámetro a la altura del pecho \pm Desvío estándar; DAP Mín: Menor DAP medido; DAP Max: Mayor DAP medido; Densidad: Número de árboles por hectárea; AB: Área basal; Proporción: Proporción de los árboles afectada; VOLi: Volumen medio de los árboles. Medias con letras distintas son significativamente diferentes $(p<0,05)$. Fuente: Elaboración Propia.

En cuanto al número de piezas por árbol, el raleo de P. híbrido produjo una mayor cantidad de rollos por árbol. Además, el optimizador provocó el corte de un mayor número de trozas por árbol independientemente de la especie. Para los rangos de 14 a $18 \mathrm{~cm}$ en punta fina, el optimizador de trozado cortó una mayor proporción de rollos de menor longitud que los cortados por el operador sin ayuda del sistema. Estas diferencias fueron de 4\% a 3\% superiores para largos de $250 \mathrm{~cm}$ y $315 \mathrm{~cm}$ y se redujo un $11 \%$ la cantidad de rollos de $375 \mathrm{~cm}$. Para rollos de 18 a $25 \mathrm{~cm}$ en punta fina también se apreció esta tendencia, donde el optimizador cortó $1 \%$ a $2 \%$ más rollos de $250 \mathrm{~cm}$ y $315 \mathrm{~cm}$ de largo (Tabla 4). Esta preferencia del optimizador por elaborar productos más cortos también fue observada por Labelle y Huß (2018). Estos autores encontraron que disminuyó la elaboración de trozas largas (mayores a $5 \mathrm{~m}$ ). El optimizador priorizó el corte de otros productos aserrables de menor longitud, ya que esto permitía lograr una mayor recuperación de valor. Debido a que el mercado local (Misiones, Argentina) de rollos aserrables no ofrece mayores precios para los productos más largos (Tabla 1), el optimizador prioriza la elaboración de trozas más cortas. De esta forma se incrementa el diámetro en punta fina y el valor de las trozas según la matriz de precios considerada. En situaciones donde existan diferenciales de precio positivos para largos y calidades, el optimizador tendería a buscar otra combinación de productos, ya que, el largo de las trozas tendría un peso importante en el valor total de la madera aprovechada (Tabla 1) (AKAY, 2017).

Tabla 4 - Duración de las actividades que componen el ciclo operacional.

Table 4 - Duration of the activities that make up the operational cycle.

\begin{tabular}{|c|c|c|c|c|c|c|c|c|c|c|c|c|}
\hline & \multirow{2}{*}{\multicolumn{3}{|c|}{$\begin{array}{l}\text { P. híbrido } \\
\text { OPT OFF }\end{array}$}} & \multirow{2}{*}{\multicolumn{3}{|c|}{$\begin{array}{c}\text { P. híbrido } \\
\text { OPT ON }\end{array}$}} & \multirow{2}{*}{\multicolumn{3}{|c|}{$\begin{array}{l}\text { P. taeda } \\
\text { OPT ON }\end{array}$}} & \multirow{2}{*}{\multicolumn{3}{|c|}{$\begin{array}{c}\text { P. taeda } \\
\text { OPT OFF }\end{array}$}} \\
\hline & & & & & & & & & & & & \\
\hline & $\bar{X}(\mathbf{s})$ & $s(s)$ & sig & $\bar{X}(\mathbf{s})$ & $s(s)$ & sig & $\bar{X}(\mathbf{s})$ & $s(s)$ & sig & $\bar{X}(\mathbf{s})$ & $s(s)$ & sig \\
\hline Búsqueda & 14,0 & 2,2 & $a$ & 15,7 & 1,8 & $a$ & 12,3 & 1,6 & $\mathrm{~b}$ & 12,6 & 0,9 & $\mathrm{~b}$ \\
\hline Volteo & 5,3 & 0,5 & a & 6,1 & 0,3 & a & 6,5 & 0,4 & $b$ & 6,6 & 0,8 & $b$ \\
\hline Trozado & 20,4 & 2,4 & a & 18,4 & 2,2 & a & 21,4 & 1,0 & $b$ & 22,5 & 2,2 & $b$ \\
\hline Liberación & 2,0 & 0,3 & a & 1,9 & 0,5 & a & 2,4 & 0,7 & $\mathrm{~b}$ & 2,3 & 0,4 & $b$ \\
\hline Vuelta a “0” & 0,3 & 0,5 & a & 0,6 & 0,5 & a & 0,6 & 0,3 & a & 0,4 & 0,3 & a \\
\hline Parada & 3,5 & 2,2 & a & 34,0 & 3,4 & a & 2,8 & 4,2 & a & 3,0 & 3,0 & $a$ \\
\hline Tiempo total & 45,6 & 4,3 & a & 46,6 & 4,5 & a & 46,0 & 3,2 & a & 47,3 & 4,6 & $a b$ \\
\hline Trozas por árbol & 4,08 & 0,07 & a & 4,45 & 0,14 & $b$ & 4,13 & 0,09 & $b$ & 4,03 & 0,08 & a \\
\hline Volumen individual (mª́rb) & 0,31 & 0,02 & a & 0,29 & 0,02 & a & 0,32 & 0,03 & $b$ & 0,33 & 0,02 & $b$ \\
\hline
\end{tabular}

$\bar{X}$ : Promedio (s). s: Desvío estándar (s). Medias con letras distintas son significativamente diferentes $(\mathrm{p}<0,05)$. Fuente: Elaboración Propia.

No hubo diferencias significativas en cuanto al porcentaje de madera aprovechada, tanto para el factor especie como para el uso del optimizador de trozado. Los árboles cosechados generaron entre un $72,73 \%$ ( $P$. taeda OPT OFF) y un $74,87 \%$ (P. híbrido OPT ON) de madera con destino aserrable de alto valor, y entre un 23,32\% (P. taeda OPT OFF) a 25,31\% (P. hibrido OPT ON) de madera destinada a trituración. La proporción de volumen remanente correspondió a trozas defectuosas, 
que no cumplieron con ningún estándar industrial y fueron desechadas en el campo $(1,85 \%$ en promedio). A pesar de que el volumen individual e intensidad de raleo fuera menor en P. híbrido, el aprovechamiento porcentual de la madera aserrable resultó similar respecto de árboles más grandes en P. taeda.

Las actividades de búsqueda de los árboles y trozado del fuste representaron más del $70 \%$ del tiempo empleado por el harvester. Esta proporción fue inferior al $84 \%$ observado por Niemistö et al., (2012) en bosques mixtos. Las otras actividades del ciclo tuvieron menor incidencia en el tiempo operacional total. Hubo diferencias significativas en el tiempo de desrame y trozado entre especies: en $P$. taeda la proporción de tiempo fue superior debido a la mayor cantidad y grosor de ramas respecto del P. híbrido. El tiempo de búsqueda fue significativamente diferente entre especies, superior en P. híbrido debido a la menor intensidad del raleo. El tiempo de volteo fue significativamente mayor en $P$. taeda, debido a que el operario realizó dos cortes para lograr el apeo de los árboles de mayor diámetro, no así para la P. híbrido donde realizó el volteo con un solo corte. Para los elementos del ciclo comprendidos en parada productiva y vuelta a " 0 ", no se encontraron diferencias significativas, tanto entre especies como por el uso del optimizador (Tabla 4).

Tanto la especie como la optimización afectaron significativamente a la productividad efectiva, al igual que la covariable volumen individual (VOLi). El P. híbrido tuvo una productividad 1,4\% mayor, mientras que el uso del optimizador resultó en una productividad 1,3\% superior. Es de destacar que cosechar P. híbrido resultó más productivo a pesar de su menor volumen individual y menor intensidad de raleo.

La mayor productividad efectiva observada en P. híbrido se debe principalmente a la forma y menor grosor de ramas, que facilitan el trabajo del cabezal y requieren un menor tiempo de desrame y trozado (LABELLE; BERGEN; WINDISCH, 2017). La mayor productividad observada al utilizar el optimizador se explica porque el sistema de control decide instantáneamente el producto a trozar mientras que el operario sin asistencia no responde con la misma velocidad ante la decisión de trozado. Este incremento de la productividad resulta inferior al $12 \%$ observado por Labelle y Huß (2018) al aplicar la optimización de trozado en Picea abies L. El empleo de un sistema de optimización para asistir al operario debería reducir la cantidad de decisiones que este tiene que tomar cuando realiza los movimientos de la máquina y específicamente en el momento del corte (KARHA et al., 2017). Sin embargo, este efecto puede verse reducido si la secuencia de productos elegida por el optimizador varía entre árboles, dificultando al operario anticipar los movimientos de la grúa durante la clasificación de los productos (LABELLE; BERGEN; WINDISCH, 2017).

El costo horario de operación del harvester fue de 149,65 US\$/h. Tanto la especie como la optimización afectaron de forma significativa al costo de producción. La cosecha de P. taeda fue $0,07 \mathrm{US} \$ / \mathrm{m}^{3}$ más costosa que para P. híbrido, y la aplicación del optimizador resultó menos costosa con una diferencia de 0,07 US $\$ / \mathrm{m}^{3}$. Aunque la interacción de factores no fue significativa, el efecto aditivo entre el P. híbrido OPT ON y P. taeda OPT OFF alcanzó una diferencia de costo de $0,14 \mathrm{US} \$ / \mathrm{m}^{3}$. El costo de producción fluctuó entre 5,27 US $\$ / \mathrm{m}^{3}$ y 5,41 US $\$ / \mathrm{m}^{3}$. Estos valores son comparables a los calculados por Mac Donagh et al., (2013b), analizando la influencia de la intensidad de extracción en operaciones de raleo en la provincia de Misiones, y ligeramente superiores a los obtenidos por Seixas y Batista (2014) para harvesters sobre orugas realizando tala rasa en Brasil.

En cuanto al valor total de la madera puesta en la industria $\left(\mathrm{US} \$ / \mathrm{m}^{3}\right)$, el factor especie no resultó significativo, al igual que la interacción de los factores. El P. híbrido alcanzó un valor unitario similar al $P$. taeda a pesar su menor intensidad de raleo y menor volumen medio de los árboles. Esto se debe a que las características favorables de forma del fuste del P. híbrido generaron un efecto positivo para la recuperación de valor.

En cambio, el factor optimización resultó significativo junto con la covariable VOLi, la cual aisló satisfactoriamente el efecto de las diferencias en el volumen individual de las parcelas. El test de Tukey separó las medias del factor optimización resultando una diferencia de 0,44 US $\$ / \mathrm{m}^{3}(2 \%)$, a favor del uso del optimizador de trozado a bordo del harvester (Tabla 5). Esta ganancia de valor es muy similar al 3\% encontrado por Labelle y Huß (2018) para la cosecha de Picea abies L., y al 3,3\% reportado por Walsh (2012) en una tala rasa de P. radiata. Posiblemente las mayores diferencias obtenidas por estos autores se deban a la diferenciación de precios de los productos por largos y calidades, y al efecto del volumen individual medio de los árboles. En el contexto de segundos raleos, con un volumen medio inferior a $0,3 \mathrm{~m}^{3}$, obteniendo menos de 3 trozas aserrables por árbol, con 
Kimmich, Hildt e Mac Donagh. - Recuperación de valor con harvester

aplicando tecnología de optimización de trozado

Tabla 5 - Parámetros de productividad, costo y valor.

Table 5 - Parameters of productivity, cost and value.

\begin{tabular}{|c|c|c|c|c|c|c|}
\hline Factor especie & $\begin{array}{c}\text { Vol i. } \\
\text { (mª́rb) }\end{array}$ & $\begin{array}{c}\text { PEF } \\
\left.\text { ( } m^{3} / h \text { ef. }\right)\end{array}$ & $\begin{array}{c}\text { COSTO } \\
\left(\mathrm{US} \$ / \mathrm{m}^{3}\right)\end{array}$ & $\begin{array}{c}\text { Valor bruto } \\
\left(\mathrm{US} \$ / \mathrm{m}^{3}\right) \\
\end{array}$ & $\begin{array}{c}\text { Valor bruto* } \\
\left(\mathrm{US} \$ \mathrm{~m}^{3}\right)\end{array}$ & $\frac{\text { Margen** }}{\left(\mathrm{US} \$ / \mathrm{m}^{3}\right)}$ \\
\hline P. híbrido & $0,301 \mathrm{a}$ & $28,05 \mathrm{~b}$ & $5,32 \mathrm{a}$ & $23,22 \mathrm{a}$ & 26,23 a & $17,61 \mathrm{a}$ \\
\hline P. taeda & $0,325 \mathrm{~b}$ & $27,66 \mathrm{a}$ & $5,39 \mathrm{~b}$ & $23,26 \mathrm{a}$ & $26,55 \mathrm{~b}$ & $17,93 \mathrm{a}$ \\
\hline \multicolumn{7}{|l|}{$\begin{array}{c}\text { Factor } \\
\text { optimización }\end{array}$} \\
\hline OPT ON & $0,305 a$ & $28,03 \mathrm{~b}$ & $5,32 a$ & $23,46 b$ & $26,63 \mathrm{~b}$ & $18,16 b$ \\
\hline OPT OFF & $0,321 \mathrm{~b}$ & $27,68 \mathrm{a}$ & $5,39 \mathrm{~b}$ & $23,02 \mathrm{a}$ & $26,23 \mathrm{a}$ & $17,61 \mathrm{a}$ \\
\hline \multicolumn{7}{|l|}{ Combinación } \\
\hline P. híbrido OPT ON & 0,294 a & $28,32 \mathrm{~b}$ & $5,27 a$ & $23,48 a$ & $26,51 \mathrm{~b}$ & $18,26 b$ \\
\hline P. híbrido OPT OFF & $0,308 \mathrm{a}$ & $27,79 a b$ & $5,37 a b$ & $22,95 \mathrm{a}$ & $26,11 a$ & $17,42 \mathrm{a}$ \\
\hline P. taeda OPT ON & $0,316 a b$ & $27,75 a b$ & $5,38 a b$ & $23,44 a$ & $26,74 \mathrm{~b}$ & $18,06 a b$ \\
\hline P. taeda OPT OFF & $0,334 \mathrm{~b}$ & $27,57 \mathrm{a}$ & $5,41 \mathrm{~b}$ & $23,09 \mathrm{a}$ & $26,36 a b$ & $17,81 a b$ \\
\hline
\end{tabular}

* Valor bruto de los productos con destino aserrable. **Margen neto antes de impuestos. Medias con letras distintas son significativamente diferentes $(p<0,05)$. Fuente: Elaboración Propia.

un conjunto de productos reducido y valorado únicamente en base a sus diámetros en punta fina, la obtención de un $2 \%$ de ganancia resulta ser un excelente resultado.

El valor (US\$/ $\mathrm{m}^{3}$ ) de los productos aserrables no presentó diferencias significativas entre especies ni interacción entre la especie y el nivel de optimización. Para el factor optimización el test de Tukey indicó que la optimización del trozado genera una diferencia significativamente positiva del orden de 0,40 US $\$ / \mathrm{m}^{3}$ de productos aserrables. El análisis demuestra que esta ganancia de valor en los productos aserrables explica el $90 \%$ del valor total generado por el optimizador, que fue de $0,44 \mathrm{US} \$ / \mathrm{m}^{3}$. Esto indica que el mayor potencial de ganancia de valor se encuentra en la correcta elección de las piezas aserrables. En este estudio el optimizador consiguió generar un surtido de productos aserrables que incrementó el valor total de la madera cosechada.

El margen antes de impuestos (MAI) no presentó diferencias significativas entre especies, ni interacción entre la especie y el nivel de optimización. La optimización de trozado generó una ganancia estadísticamente significativa de 0,55 US $\$ / \mathrm{m}^{3}$. El optimizador agregó valor a la madera cosechada, y además mejoró la productividad del harvester, reduciendo el costo de cosecha y generando una diferencia en el MAI positiva a favor del uso del optimizador. Estos resultados son concordantes con Walsh (2012), que encontró una reducción de costos de cosecha por aumento de productividad del harvester, y simultáneamente ganancia del valor de la madera cosechada. El hecho que el tamaño menor cosechado en P. híbrido no haya afectado de forma negativa a la MAI, significa que la forma del fuste y tamaño de ramas juega un papel fundamental en la generación de valor por parte del optimizador, y que afecta a las decisiones de trozado.

\section{CONCLUSIONES}

La aplicación del optimizador de trozado generó una ganancia en el valor general. El apoyo a las decisiones con esta tecnología posibilitó aumentar la productividad y disminuir los costos significativamente, contribuyendo tambin a un mejor aprovechamiento de los rboles cosechados.

Por otra parte, el efecto de la mejor forma del fuste del P. híbrido produjo un efecto positivo para el optimizador, compensando el menor volumen individual de estos árboles respectos a los de $P$. taeda. El optimizador se vio potenciado por la forma, conicidad y ramas pequeñas del P. hibrido por encima de P. taeda, y esto posibilitó no solo mayores valores cosechados por árbol con plantas volumétricamente más pequeñas, sino que mejoró la productividad del harvester.

En este estudio el optimizador no produjo un aumento del volumen total aprovechado. Sin embargo, la selección correcta de piezas aserrables fue la clave que permitió incrementar el valor recuperado. La combinación de costos más bajos en P. híbrido y aumento del valor de la madera cosechada generó márgenes de ganancias antes de impuestos superiores a los demás tratamientos en una magnitud de $4,8 \%$ con respecto a $P$. taeda sin optimización. 


\section{REFERENCIAS}

ACKERMAN, P.; BELBO, H.; ELIASSON, L.; JONG, A.d.; LAZDINS, A.; LYONS, J. The Cost model for calculation of forest operations costs. International Journal of Forest Engineering, v. 25, n. 1, p. 75-81, 2014.

AKAY, A. E. Potential Contribution of Optimum Bucking Method to Forest Products Industry. European Journal Forest. Engeniring, Bursa, v. 3, n. 2, p. 61-65, 2017.

KARHA, K.; ÄNÄKKÄLÄ, J.; HAKONEN, O.; PALANDER, T.; SORSA, J.; RÄSÄNEN, T.; MOILANEN, T. Analyzing the antecedents and consequences of manual log bucking in mechanized wood harvesting. Mechanics, Materials Science \& Engineering, v. 12, n. 1, p. 1-15, 2017.

LABELLE, E. R.; HUSS, L. Creation of value through a harvester on-board bucking optimization system operated in a spruce stand. Silva Fennica, Helsinki, v. 52, n. 3, p. 1-22, 2018.

LABELLE, E. R.; BERGEN, R.; WINDISCH, J. The effect of quality bucking and automatic bucking on harvesting productivity and product recovery in a pine-dominated stand. European Journal of Forest Research, v. 136, n. 4, p. 639-652, 2017.

LU, K.; BI, H.; WATT, D.; STRANDGARD, M.; LI, Y. Reconstructing the size of individual trees using log data from cut-to-length harvesters in Pinus radiata plantations: a case study in NSW, Australia. Journal of Forestry Research, v. 29, n. 1, p. 13-33, 2018.

MAC DONAGH, P. M.; MULAWKA, J. A.; FRIEDL, R. A.; ZADERENKO, C.; ALEGRANZA, D. A. Productividad y costos de pequeños "harvester" con oruga, operando en plantaciones de Pinus spp. en el noreste argentino. Scientia Forestalis, Piracicaba, v. 42, n. 99, p. 4C25-434, 2013 a.

MAC DONAGH, P. M.; HILDT, E.; FRIEDL, R. A.; ZADERENKO, C.; ALEGRANZA, D. A. Influencia de la intensidad de raleos en la performance de un harvester de ruedas en el noreste Argentino. Floresta, Curitiba, v. 43, n. 4, p. 653-662, 2013 b.

MALINEN, J.; TEPPO, P.; HARRI, K.; TAPIO, W.; ERKKI, V. Timber assortment recovery models for southern Finland, Baltic Forestry, Kaunas, v. 16, n. 1, p. 102-112, 2010.

MURPHY, G. E.; ACUNA, M. A.; DUMBRELL, I. Tree value and log product yield determination in radiata pine (Pinus radiata) plantations in Australia: comparisons of terrestrial laser scanning with a forest inventory system and manual measurements. Canadian Journal of Forest Research, Otawa, v. 40, n. 11, p. 2223-2233, 2010.

NAKAHATA, C.; ARUGA, K.; SAITO, M. Examining the Optimal Bucking Method to Maximize Profits in Commercial Thinning Operations in Nasunogahara Area, Tochigi Prefecture, Japan. Croatian Journal of Forestr Engineering, Zagreb, v. 35, n. 1, p. 45-61, 2014.

NIEMISTÖ, P.; KORPUNEN, H.; LAURÉN, A.; SALOMÄKI, M.; UUSITALO, J. Impact and Productivity of Harvesting while Retaining Young Understorey Spruces in Final Cutting of Downy Birch. Siva Fennica, Helsinki, v. 46, n. 1, p. 81-97.

PEÑA ZUBIATE, C. A; PINEDO, D. M. Atlas de suelos de la República Argentina. Buenos Aires: Secretaría de Agricultura, Ganadería y Pesca (SAGyP). Buenos Aires: Instituto Nacional de Tecnología Agropecuaria / INTA, 1990. $600 \mathrm{p}$.

SEIXAS, B.; BATISTA, J. L. F. Comparação técnica e econômica entre harvesters de pneus e com máquina base de esteiras, Ciência Florestal, Santa Maria, v. 24, n. 1, p. 185-191, 2014.

SKOGFORSK. StanForD “Classic” Documents. 2016. Disponible en: < http://www.skogforsk.se/english/projects/ stanford/ >.Acesso en: 20 out. 16

SOIL SURVEY STAFF. Soil Taxonomy. Washington, D.C: United States Department of Agriculture, 1999. 871 p. (Handbook, 436)

UUSITALO, J. Introduction to Forest Operations and Technology. Tampere: JVP Forest Systems Oy, 2010, 287 p.

WALSH, D. Quantifying the value recovery improvement using a harvester optimiser. CRC for Forestry, Tasmania, Australia, v. 26, p. 1-4, 2012.

Recebido em: 23/02/2018

Aceito em: 18/09/2018

Sci. For., Piracicaba, v. 47, n. 122, p. 216-223, jun. 2019

DOI: doi.org/10.18671/scifor.v47n122.03 